\title{
A DETERMINATION OF MAGNETIC FIELDS OF T TAU AND Ae/Be HERBIG STARS USING THE PARAMETERS OF THEIR LINEAR POLARIZATION
}

\author{
Yu. N. Gnedin, M. A. Pogodin \\ Central Astronemical Observatory of \\ the USSR Academy of Sciences \\ Pulkovo, 196140 Leningrad \\ USSR
}

\author{
N. P. Red'kina \\ Institute of Astrophysics \\ Tadzhic Academy of Sciences \\ Dushanbe \\ USSR
}

\begin{abstract}
Attempts of applying stellar polarization spectra to evaluation of magnetic fields of some rypes of stars are described.
\end{abstract}

Gnedin and Silant'ev (1980) proposed a method for determining stellar magnetic fields using linear polarization spectra of stars. The method was based on the effect of the Faraday rotation of the plane of polarization produced by the electron scattering in a magnetized circumstellar shell. The asymmetry of spatial distribution of the magnetic field should lead to linear polarization. Gnedin and Silant'ev gave spectral curves, calculated for spherical and ellipsoidal envelopes with different values of the dipole magnetic field $B$, optical thickness of the envelope $\tau_{\mathrm{e}}$ and the inclination angle of the axis to the line-of-sight $\theta_{\mathrm{m}}$. The shape of these curves has characteristic maximum. Its position and polarization degree depends on the envelope form and the set of parameters $B, \tau_{e}$, and $\theta_{m}$.

Attempts of using the proposed method were made for determination of magnetic fields of $\mathrm{T} \mathrm{Tau}$ and $\mathrm{Ae} / \mathrm{Be}$ Herbig stars. These have gaseous envelopes and intrinsic polarization with the maximum in the visible spectral range. Moreover, the existence of magnetic fields of these stars predicted in a series of theoretical works (see, for example, Gnedin and Red'kina, 1984). One of the main difficulties in applying the method was the multicomponent nature of the observed linear polarization of these objects. Its parameters are influenced not only by the electron scattering in the gaseous envelope but also by a scattering in a) the circumstellar dust envelope b) a local nebula, associated with the star and c) interstellar medium with a highly inhomogeneous matter distribution at small galactic latitudes. The important problem was to correctly distinguish the component belonging to the gaseous envelope.

Firstly, this method was employed for the star T Tau (Gnedin and Red'kina, 1984). An assumption was used that the intrinsic polarization of $T$ Tau is mainly caused by eiectron scattering, and the effect of the dust component could be ignored. This supposition was based on a discovery of a cool satellite of the star which can explain the IR excess in the $T$ Tau 


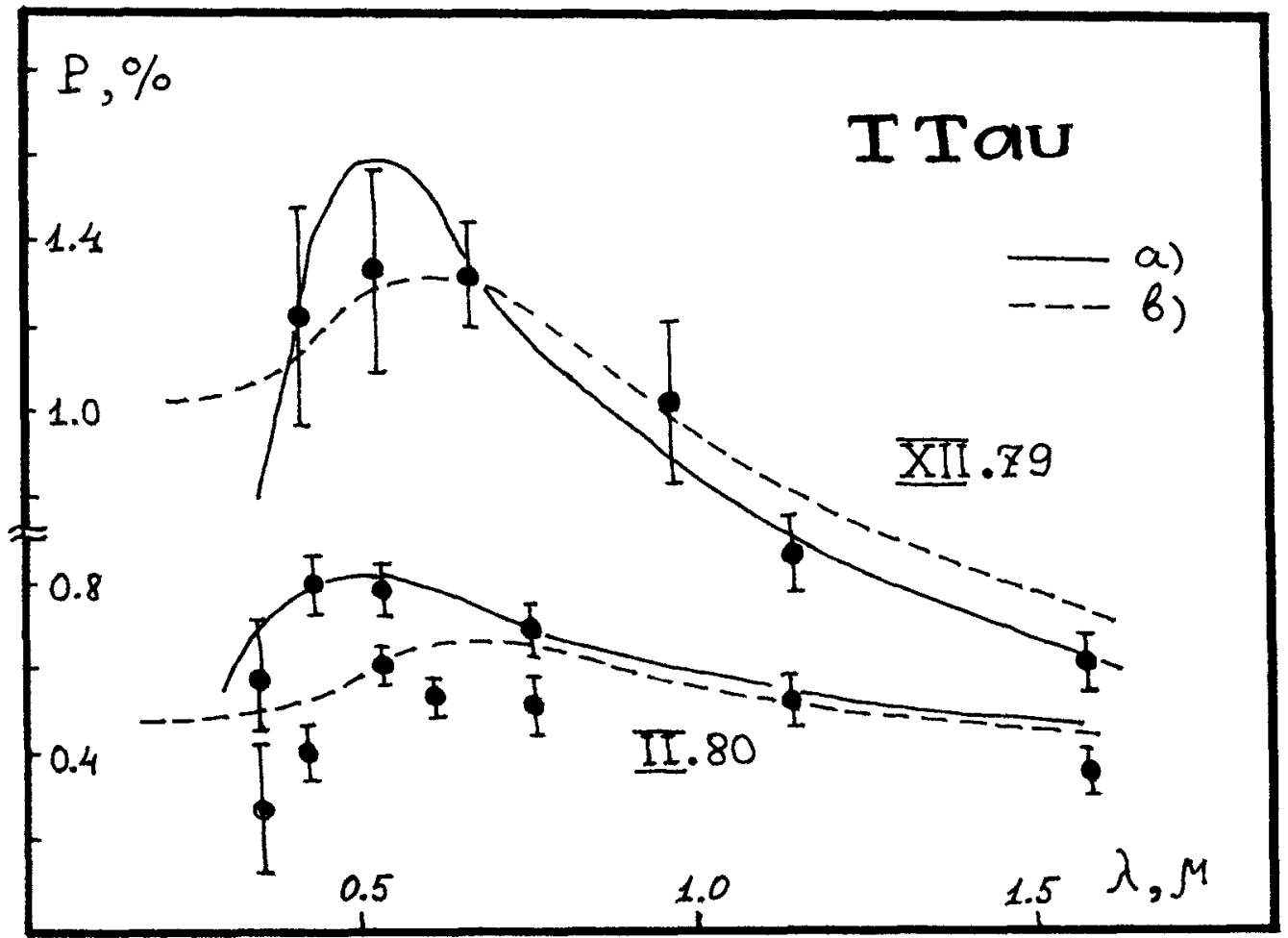

Figure 1. Comparison of the observed spectre of the intrinsic linear polarization of $T$ Tau with theoretical curves. The values of the model parameters are:

December 1979:a)sphertcal model, $B=250$ Gauss, $\tau_{e}=0.20$

b)ellipsoidal model with the ratio of principal axes $0.25, B=1500$ Gauss, $\tau_{e}=0.14$.

February $1980: a)$ spherical model, $B=400$ Gauss, $\tau_{e}=0.13$

b) ellipsoidal model, $B=1200$ Gauss, $\tau_{e}=0.07$.

spectrum (Dyck and Simon, 1982). Thus, this can justify the rejection of the hypothesis of the existence of a considerable dust envelope round $T$ Tau. $A$ comparison of the $T$ Tau polarization spectrum free from interstellar component with calculated curves, gave a value of the magnetic field of the star B varying from 100 to 400 Gauss for the spherical model and from 300 to 1500 Gauss for the ellipsoidal model.

The evaluations of the fields of RY Tau, SU Aur, RW Aur and DI Cep were made similarly. A strong polarimetric variability of these objects (Figure 2) can be considered as an additional argument in favor of the mechanism of electron scattering producing the polarization. Their magnetic fields also attain the values of the order $10^{2}-10^{3}$ Gauss.

In the work by Gnedin and Pogodin (1985) devoted to a determination of magnetic fields of Ae/Be Herbig stars, an attempt was made to separate the gaseous and dust components of intrinsic linear polarization. Independent evaluations of $\tau_{\mathrm{e}}$ obtained from the 


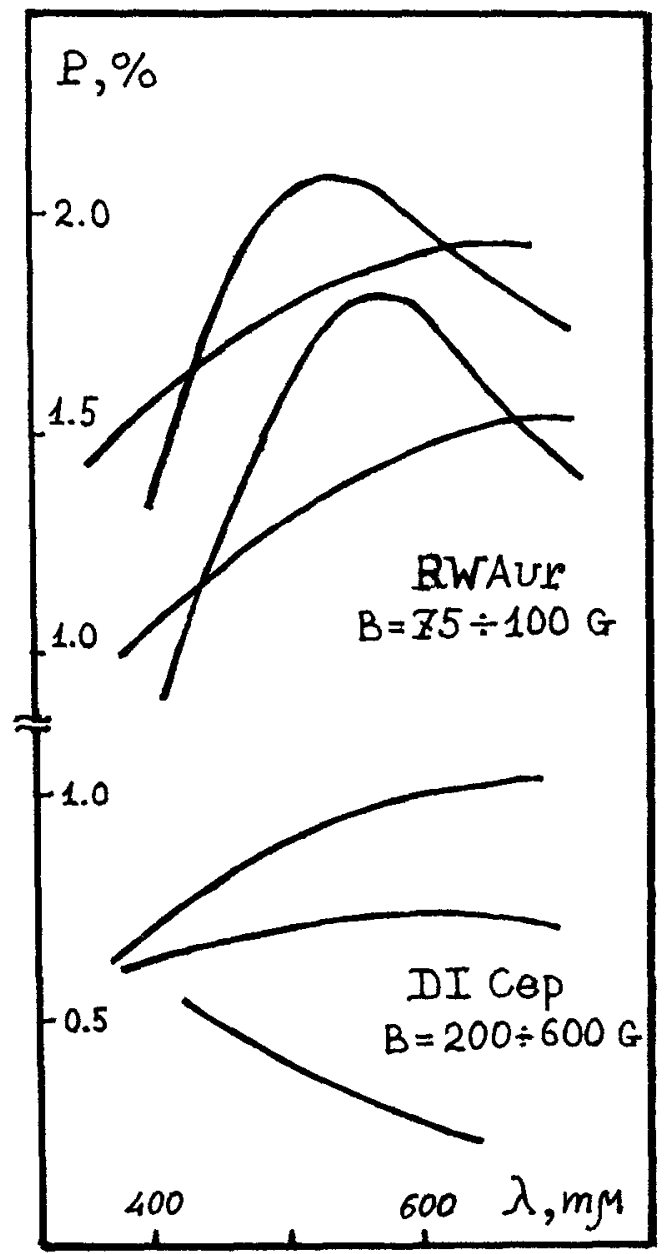

Pigure 2. Variability of the apectrum of the intringic inear polarisation of some T Tau stara.

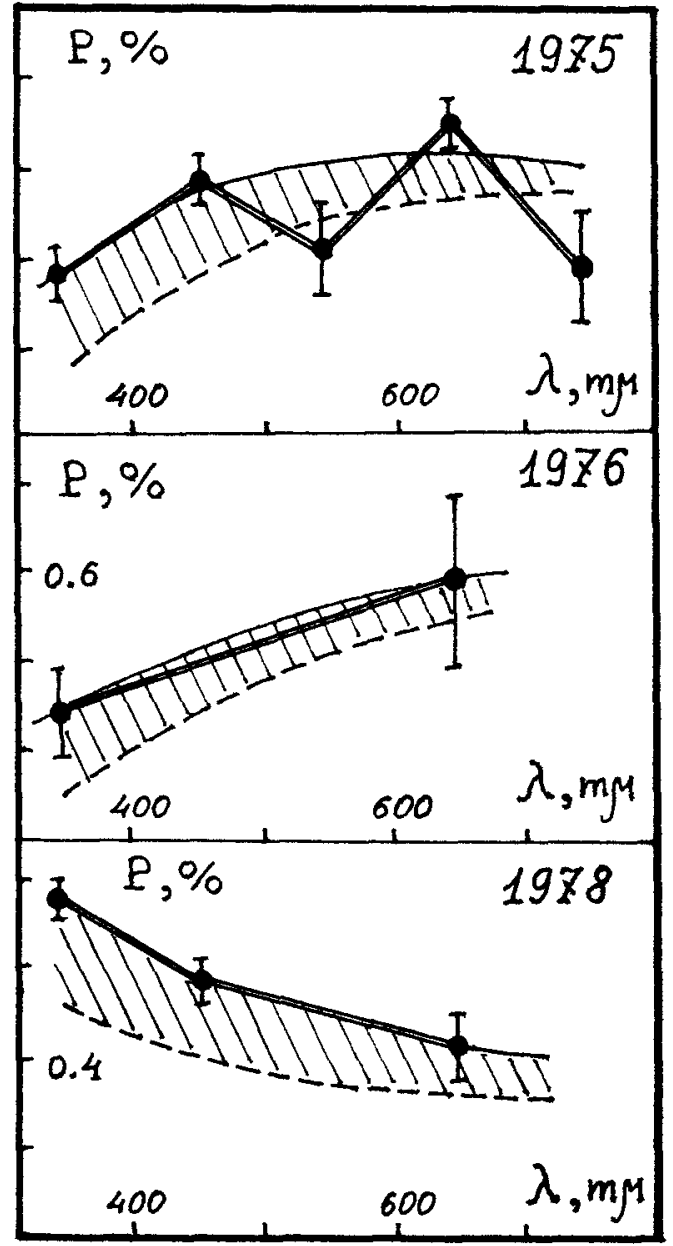

Figure 3. Separation of the gaseous and duet $\left(P(\lambda) \sim \lambda^{-1}\right)$ components of the intrinsic incar polarieation of HD 200775. The dust component is shaded. The values of the model parane ters are: $\tau_{e}=0.17$ a) 1975:B=110 Gauss, $\theta_{m}=70^{\circ}$ b) 1976:B=110 Gauss, $\theta_{m}=60^{\circ}$ c) $B=550$ Gause, $\theta_{m}=75^{\circ}$. 
observed emission Balmer jump (see Garrison, 1978) were also employed. The results of a separation of the components in polarization spectrum of the star HD 200775 are given in Figure 3. Polarimetric variability can be explained in terms of variation of the magnetic field $(\mathrm{B}=100$ to 500 Gauss) and a precession for the magnetic axis of the star, the dust component being assumed invariable.

\section{REFERENCES}

Dyck, H. M., Simon, T., 1982, Ap. J. Lett., 255, 103.

Garrison, L. M., 1978, Ap. J., 224, 535.

Gnedin, Yu. N., Silant'ev, N. A., 1980, Sov. Astron. J. Lett., 6, 344.

Gnedin, Yu. N., Red'kina, N. P., 1984, Sov. Astron. J. Lett., 10, 613.

Gnedin, Yu. N., Pogodin, M. A., 1985, Sov. Astron. J. Lett., 11. 37. 